\title{
Plummer-Vinson Syndrome in an African-American Woman
}

\author{
Keshav Patel ${ }^{a} \quad$ Mahmoud Kassir ${ }^{b}$ Madhav Patel ${ }^{c}$ Wesley Eichorn ${ }^{b}$ \\ aWestern Michigan University Homer Stryker MD School of Medicine, Kalamazoo, MI, USA; \\ bepartment of Family and Community Medicine, Western Michigan University Homer \\ Stryker MD School of Medicine, Kalamazoo, MI, USA; 'Georgetown University School of \\ Medicine, Washington, DC, USA
}

\section{Keywords}

Plummer-Vinson syndrome $\cdot$ Iron-deficiency anemia $\cdot$ Dysphagia $\cdot$ Esophageal web

\begin{abstract}
Plummer-Vinson syndrome (PVS) presents with the classic triad of iron-deficiency anemia, dysphagia, and esophageal webs. The mainstay treatment of PVS is iron supplementation and the dysphagia usually responds to iron supplementation before the hematologic abnormalities are corrected. This syndrome classically affects middle-aged Caucasian women and very few cases have been reported in African Americans. We present a rare case of PVS in an African-American woman. A 63-year-old woman presented with shortness of breath, dysphagia, and lightheadedness for several weeks. Chest X-ray was negative for any acute abnormalities. Initial hemoglobin was $7.0 \mathrm{~g} / \mathrm{dL}$, which improved to $7.5 \mathrm{~g} / \mathrm{dL}$ after 1 unit of packed red blood cells. She had a mean corpuscular volume of $62 \mathrm{fL}$, a ferritin level of $6 \mathrm{ng} / \mathrm{mL}$, and an iron level of $12 \mu \mathrm{g} / \mathrm{dL}$. Fecal occult blood test was negative and barium swallow revealed a proximal esophageal web. Her dysphagia did not significantly improve despite intravenous iron supplementation and esophageal web dilation. Video-fluoroscopic swallow study revealed esophageal and pharyngeal phase dysphagia with food entrapment. She was discharged with plans to follow up with a primary care physician and repeat esophagogastroduodenoscopy in 1 year. This case report highlights a rare case of PVS in an African-American woman and emphasizes the importance of maintaining a comprehensive and broad differential diagnosis.

(C) 2021 The Author(s).

Published by S. Karger AG, Basel
\end{abstract}

\section{Introduction}

Plummer-Vinson syndrome (PVS), also known as Patterson-Kelly syndrome, presents with the classic triad of iron-deficiency anemia, dysphagia, and esophageal webs [1]. Other common findings in PVS include glossitis, koilonychia, and enlargement of the spleen and 
thyroid. Iron-deficiency anemia is the most important risk factor; however, other risk factors include malnutrition, autoimmune diseases, or genetic predisposition [2]. The pathogenesis of this extremely rare condition is unclear; however, iron deficiency plays a key role, and it is hypothesized that depletion of iron-dependent oxidative enzymes causes myasthenic changes in swallowing muscles, esophageal mucosal atrophy, and formation of esophageal webs [1]. The best initial imaging study is the barium esophagogram [1]. Esophagogastroduodenoscopy (EGD) can also be done in order to visualize the esophageal web and perform esophageal web dilation. The mainstay treatment of PVS is iron supplementation and the dysphagia usually responds to iron supplementation before the hematologic abnormalities are corrected [2]. Most of the patients respond to iron supplementation and esophageal web dilation is rarely required [3]. PVS is extremely rare now due to a decrease in iron-deficiency and malnutrition. Between 1999 and 2005, there were only 28 PVS English written case reports in the literature [2]. This syndrome classically affects middle-aged Caucasian women. Even though both malnutrition and iron-deficiency anemia are common in Africa, very few cases have been reported in African Americans. We present a rare case of PVS in an African-American woman.

\section{Case Report/Case Presentation}

A 63-year-old patient with a past medical history of chronic obstructive pulmonary disease, alcohol abuse, and hepatitis $\mathrm{C}$ presented with worsening shortness of breath, dizziness, and weakness for several weeks. The shortness of breath occurred with moderate exertion and the dizziness occurred when she stood up. The patient also had worsening coughing episodes for the last week. She also had dysphagia to both solids and liquids that had progressed over the last 6 months. She had lost approximately 10 pounds in the last 6 months and her initial weight during this hospitalization was 106 pounds. She denied any fevers, chills, abdominal pain, diarrhea, hematemesis, hematuria, hematochezia, or abnormal uterine bleeding. She was homeless and noncompliant with her medications. She drinks 3 beers daily and is a smoker with a 30-pack year history. Regarding her vital signs, she was afebrile $(98.6 \mathrm{~F})$ with a heart rate of 91 beats/minute, respiratory rate of 22 breaths/minute, blood pressure of $132 / 62 \mathrm{~mm} \mathrm{Hg}$, and an oxygen saturation of $100 \%$ on room air. She was not in any acute distress. Regarding her skin, there was pallor noted bilaterally. On pulmonary exam, there were coarse breath sounds bilaterally. The rest of the physical exam was unremarkable. Her shortness of breath improved with prednisone and albuterol. Of note, she was not compliant with her chronic obstructive pulmonary disease home medications. Chest $\mathrm{X}$-ray was negative for any acute abnormalities. Complete blood count revealed a hemoglobin of $7.0 \mathrm{~g} / \mathrm{dL}$ which improved to $7.5 \mathrm{~g} / \mathrm{dL}$ after 1 unit of packed red blood cells. Mean corpuscular volume was $62 \mathrm{fL}$, ferritin level was $6 \mathrm{ng} / \mathrm{mL}$, iron level was $12 \mu \mathrm{g} / \mathrm{dL}$, transferrin was elevated at $505 \mathrm{mg} / \mathrm{dL}$, and total iron-binding capacity was elevated at $641 \mu \mathrm{g} / \mathrm{dL}$ (shown in Table 1). Fecal occult blood test was negative and barium swallow revealed a proximal esophageal web (shown in Fig. 1a, b).

EGD was performed and the esophageal web was dilated. Despite intravenous iron supplementation (total of $800 \mathrm{mg}$ iron sucrose) and esophageal web dilation, the patient's dysphagia did not significantly improve during her hospital stay. Video-fluoroscopic swallow study revealed esophageal and pharyngeal phase dysphagia with food entrapment. We recommended a thin liquid diet; however, she was homeless and did not have the resources to adhere to this type of diet. We advised placement in a shelter or group home, but the patient refused. She was discharged with plans to follow up with a primary care physician in a few days and repeats the EGD in 1 year. Since discharge, the patient has been lost to follow-up.

\section{Karger'}


Table 1. Initial lab values

\begin{tabular}{lll}
\hline & Lab values & Reference range \\
\hline White blood cell count & 6.1 & $4.0-11.0 \times 10^{9} / \mathrm{L}$ \\
Red blood cell count & 4.16 & $4.2-5.5 \times 10^{12} / \mathrm{L}$ \\
Hemoglobin & 7 & $12.0-16.0 \mathrm{~g} / \mathrm{dL}$ \\
Hematocrit, \% & 25.8 & $36.0-47.0$ \\
MCV & 62 & $80-99 \mathrm{fL}$ \\
MCH & 16.8 & $25.0-35.0 \mathrm{pg} / \mathrm{cell}$ \\
MCHC & 27.1 & $32.0-36.0 \mathrm{~g} / \mathrm{dL}$ \\
RCW & $19.6 \%$ & $<15.0 \%$ \\
Platelet & 395 & $140-440 \times 10^{9} / \mathrm{L}$ \\
Ferritin & 6 & $15-150 \mathrm{ng} / \mathrm{mL}$ \\
Absolute reticulocyte count & 49.4 & $23-93 \times 10^{9} / \mathrm{L}$ \\
Reticulocyte count percentage, \% & 1.2 & $0.5-1.5$ \\
Reticulocyte hemoglobin & 15.5 & $28.6-36.3 \mathrm{pg} / \mathrm{cell}$ \\
Immature reticulocyte fraction & 19.9 & $2.3-15.9 \%$ \\
Iron & 12 & $60-135 \mu \mathrm{g} / \mathrm{dL}$ \\
Transferrin & 505 & $200-360 \mathrm{mg} / \mathrm{dL}$ \\
TIBC & 641 & $254-457 \mu \mathrm{g} / \mathrm{dL}$ \\
Iron saturation, \% & 2 & $15-50$ \\
\hline
\end{tabular}

MCV, mean corpuscular volume; MCH, mean corpuscular hemoglobin; MCHC, MCH concentration; RCW, red cell distribution with; TIBC, total iron-binding capacity.
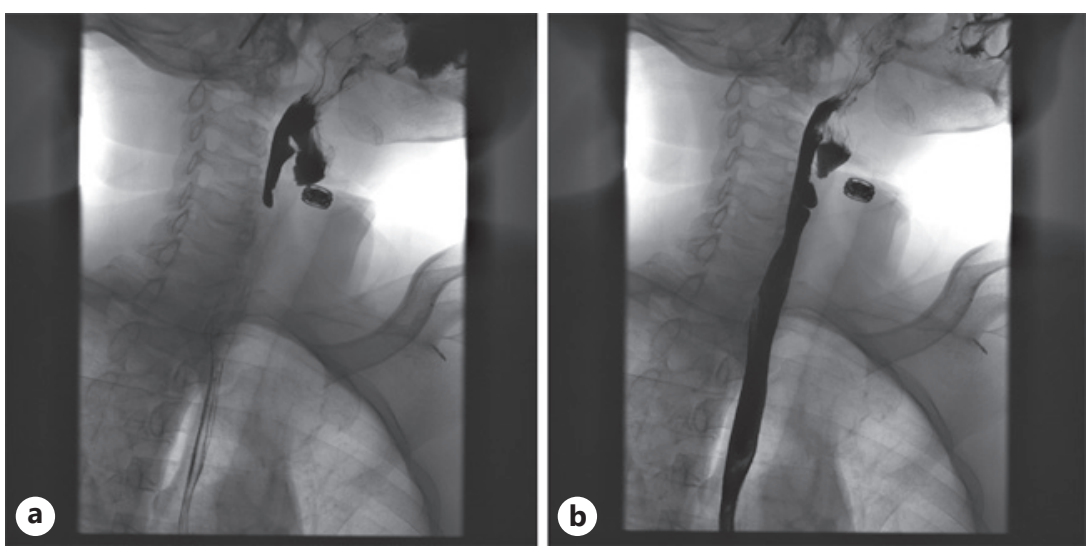

Fig. 1. Barium swallow revealed a proximal esophageal web. In image (a), we can see the barium in the upper esophagus. In image (b), the barium extends to the distal esophagus.

\section{Discussion/Conclusion}

Our patient presented with shortness of breath, dysphagia, and cough. She was found to have iron-deficiency anemia and was diagnosed with PVS after the barium esophagogram revealed a proximal esophageal web. Esophageal webs usually occur in the proximal 4-5 cm of the esophagus and webs that are $<12 \mathrm{~mm}$ can cause dysphagia [4]. For most patients, the dysphagia responds to iron supplementation. In patients who continue to experience dysphagia, 
esophageal web dilation can be performed. Despite intravenous iron supplementation for a total of $800 \mathrm{mg}$ iron sucrose and esophageal web dilation, our patient's dysphagia did not improve significantly.

Video-fluoroscopic swallow study revealed esophageal and pharyngeal phase dysphagia with food entrapment, and she was placed on a thin liquid diet. Our patient was homeless and did not have the resources to adhere to a thin liquid diet. We recommended placement, however the patient refused.

Our patient most likely had iron-deficiency anemia due to a poor oral intake. She was homeless and did not have access to healthy foods. She had also lost approximately 10 pounds in thelastfew months. During hospitalization, we consulted a registered dietician and encouraged the patient to eat well-balanced and nutritious meals. Interestingly, our patient did not have a deficiency in any other minerals or vitamins.

PVS can be difficult to diagnose since it is extremely rare and the symptoms of anemia may dominate the clinical picture. Our patient had the classic triad of PVS: dysphagia, iron-deficiency anemia, and an esophageal web. PVS is precancerous, and early recognition is therefore crucial. PVS has an excellent prognosis in most patients; however, 3-15\% of patients with PVS develop cancer and their prognosis is significantly worse [2]. The most common cancer to develop in PVS patients is squamous cell carcinoma of the pharynx and esophagus. Our patient had a 30-pack year smoking history and has an increased risk of developing cancer. Our patient was discharged with plans to follow up with her primary care physician in a few days and repeat EGD in 1 year.

Between 1999 and 2005, there were only 28 English language PVS case reports in the literature [2]. We searched PubMed with the search string ("Plummer-Vinson Syndrome" [Mesh] OR "plummer vinson" [tiab] OR "patterson's syndrome" [tiab] OR "Patterson syndrome" [tiab] OR "pattersons syndrome" [tiab] OR "Patterson Kelly" [tiab] OR "Patterson-kelly" [tiab] OR "Kelly syndrome" [tiab] OR "kellys syndrome" [tiab] OR "kelly's syndrome" [tiab] OR "Patterson brown Kelly" [tiab] OR "Patterson-brown-kelly" [tiab]) and limited the search to case reports published since 2011. This resulted in merely 44 entries, with just 5 entries reporting a patient that is African American or Black. Although this search may not include every single case of PVS in an African American, the search results further emphasize the uniqueness of this case report. By presenting this extremely rare case of PVS in an African-American woman, we hope to emphasize the importance of maintaining a comprehensive and broad differential diagnosis. We also hope to further emphasize the importance of further workup in patients with dysphagia, especially when accompanied by iron-deficiency anemia.

\section{Acknowledgements}

Juli McCarroll, MA, MLIS, and AHIP. Informationist/Medical Librarian. She is aware and in agreement with her name appearing in this manuscript in case of publication.

\section{Statement of Ethics}

The patient has given their written informed consent to publish their case (including publication of images). This study protocol was reviewed and the need for approval was waived by the Western Michigan University School of Medicine IRB office.

\section{Karger's}




\section{Case Reports in Gastroenterology}

\section{Conflict of Interest Statement}

The authors have no conflicts of interest to declare. K.P. and M.P. are siblings (not a conflict of interest).

\section{Funding Sources}

This study was not receiving any funds.

\section{Author Contributions}

K.P. and M.P. wrote the initial draft. M.K. and W.E. edited the manuscript and provided intellectual input.

\section{References}

1 Phatak S, Redkar N, Patil MA, Kuwar A. Plummer-Vinson syndrome. BMJ Case Rep. 2012 July;2012: bcr2012006403.

2 Novacek G. Plummer-Vinson syndrome. Orphanet J Rare Dis. 2006 Sep;1:36.

3 Gade AK, Pacheco L. A rare case of plummer-vinson syndrome. Cureus. 2019 Dec;11(12):e6463.

4 Karthikeyan P, Aswath N, Kumaresan R. Plummer vinson syndrome: a rare syndrome in male with review of the literature. Case Rep Dent. 2017 Aug;2017:6205925. 